\title{
Challenges associated with the management of gynecological cancers in a tertiary hospital in South East Nigeria
}

This article was published in the following Dove Press journal:

International Journal of Women's Health

24 January 2014

Number of times this article has been viewed

\section{Chukwuemeka Anthony lyoke'}

George Onyemaechi Ugwu' Euzebus Chinonye Ezugwu' Frank Okechukwu Ezugwu ${ }^{2}$ Osaheni Lucky Lawani ${ }^{3}$

Azubuike Kanayo Onyebuchi $^{3}$

'Department of Obstetrics and Gynaecology, University of Nigeria Teaching Hospital, Ituku-Ozalla, Enugu, ${ }^{2}$ Department of Obstetrics and Gynaecology, Enugu State University Teaching Hospital, Park Lane, Enugu, ${ }^{3}$ Department of Obstetrics and Gynaecology, Federal Teaching Hospital, Abakaliki, Ebonyi State, Nigeria
Correspondence: Chukwuemeka Anthony lyoke

Department of Obstetrics and Gynaecology, University of Nigeria Teaching Hospital, Ituku-Ozalla, PO Box 4994, Enugu Headquarters, Enugu, Enugu State, Nigeria Email caiyoke@yahoo.co.uk
Background: There are reports of increasing incidence of gynecological cancers in developing countries and this trend increases the need for more attention to gynecological cancer care in these countries.

Objective: The purpose of this study was to describe the presentation and treatment of gynecological cancers and identify barriers to successful gynecological cancer treatment in a tertiary hospital in South East Nigeria.

Methods: This study was a retrospective longitudinal analysis of the presentation and treatment of histologically diagnosed primary gynecological cancers from 2000 to 2010 . Analysis was by descriptive and inferential statistics at the $95 \%$ level of confidence using Statistical Package for the Social Sciences version 17 software.

Results: Records of 200 gynecological cancers managed during the study period were analyzed. Over $94 \%$ of cervical cancers presented in advanced stages of the disease and received palliative/symptomatic treatment. Only $1.9 \%$ of cervical cancer patients had radical surgical intervention, and postoperative mortality from these radical surgeries was $100 \%$. Approximately $76 \%$ of patients with ovarian cancer had debulking surgery as the mainstay of treatment followed by adjuvant chemotherapy. Postoperative mortality from ovarian cancer surgery was $63 \%$. Cutting edge cytotoxic drugs were not used as chemotherapy for ovarian and chorionic cancers. Compliance with chemotherapy was poor, with over $70 \%$ of ovarian cancer patients failing to complete the prescribed courses of chemotherapy. Most patients with endometrial and vulval cancers had only surgical treatment, as compliance with follow-up for adjuvant chemotherapy or radiotherapy was poor. Functional radiotherapy facilities were not available at the center during the study period, thereby necessitating external referrals to centers hundreds of kilometers away.

Conclusion: Late presentation of cases, noncompliance with treatment regimens, lack of use of cutting edge cytotoxic drugs, the poor outcome of radical surgeries, and lack of a functional radiotherapy facility combined to create a very difficult gynecological cancer care environment at the study center.

Keywords: gynecological cancer, management challenges, cancer surgery, chemotherapy

\section{Introduction}

There is an increasing documentation of cancers, including gynecological cancers, in developing countries. ${ }^{1}$ A recent global report by the International Agency for Research on Cancer showed that gynecological cancers accounted for $25 \%$ of all new cancers diagnosed in women aged up to 65 years compared with $16 \%$ in the developed world. ${ }^{2}$ Similarly, a report on the global burden of cancer showed that developing countries accounted for 820,265 cases $(77.7 \%$ ) of global estimates for new cases of 
the commonest gynecological cancers, including cervical, corpus, and ovarian cancer, in $2009 .{ }^{1}$

This observed trend in developing countries has been attributed to several possibilities including the shift to a Western lifestyle and behaviors such as cigarette smoking, low fiber/high fat diets, and less physical activity; a high prevalence of immunosuppressing conditions such as malnutrition, tuberculosis, and human immunodeficiency virus; a high prevalence of oncogenic infections such as hepatitis B virus, human immunodeficiency virus, hepatitis $\mathrm{C}$ virus, human papilloma virus, and Helicobacter pylori. ${ }^{3}$ It has also been suggested that this trend could be, in part, due to reporting bias arising from increasing use of orthodox care occasioned by increasing health awareness at the population level in some developing countries. ${ }^{4}$

With the increasing documentation of gynecological cancers in developing countries, it would be expected that efforts at screening and management of cancers should be scaled up to meet the increasing demands for care. Unfortunately, challenges to these efforts appear to persist and a huge unmet need for funding for gynecological cancer care continues to prevail in developing countries. ${ }^{5,6}$

There have been several reports on the prevalence of gynecological cancers in South East Nigeria. ${ }^{7,8}$ Gynecological cancers have been shown to contribute overwhelmingly to gynecological mortality in our environment. ${ }^{9}$ However, we found scanty literature on the management of these cancers. This study describes the challenges in management of gynecological cancers in a major referral hospital in South East Nigeria over the last decade in order to highlight the major clinical barriers to successful outcomes in the management of gynecological cancers.

\section{Materials and methods}

This was a retrospective longitudinal analysis of the presentation and treatment of histologically diagnosed primary gynecological cancers managed at the study center. The study covered the period from January 1, 2000 to December 31, 2010. The study population included all women admitted and treated in the gynecology department of the hospital during the study period. A purposive sample of consecutive women with gynecological cancer was taken. A written application was made to the cancer registry of the study center, following which all cases of gynecological cancer seen during the study period and their biographic, clinical, and mortality data were compiled by the cancer registry. Subsequently, the histological diagnoses of these cases were retrieved from the histopathology department of the hospital.
The case notes of these selected cases were retrieved from the records department, and the records of each patient were followed up from presentation until the last records of care. Data relating to age, parity, clinical presentation, and treatment modalities, including details of surgical procedures, chemotherapy regimens, and outcomes of treatment, were abstracted and coded using Statistical Package for the Social Sciences version 17 software (IBM Corporation, Armonk, NY, USA) for Windows for analysis. Where case notes were not available or had missing data, efforts were made to obtain patient data from ward admission records and operating theater records. Mortality data were compiled from the cancer registry records, case notes, and mortuary records. Data analysis was by descriptive and inferential statistics at the $95 \%$ level of confidence. $P$-values $\leq 0.05$ were considered to be statistically significant. Ethical consent was obtained from the research ethics committee of the University of Nigeria Teaching Hospital, Enugu.

\section{Results}

The total number of histologically diagnosed cases of primary gynecological cancer obtained over an elevenyear period was 236. The records of 200 cases could be retrieved, giving a recovery rate of $84.7 \%$. Case records were available for $76.3 \%(106 / 139)$ of cases of cervical cancer, $92.6 \%(50 / 54)$ of cases of ovarian cancer; all 19 cases of endometrial cancer, ten cases of choriocarcinoma, ten cases of vulvar cancer, and four cases of leiomyosarcoma. The case record for the only case of vaginal cancer was not available. The total number of gynecological admissions during the period was 6,527. Gynecological malignancies constituted $3.6 \%$ of all gynecological admissions. Table 1 shows the distribution of gynecological cancers based on patient characteristics.

\section{Cervical cancer}

There were 139 cases of cervical cancer, constituting $2.1 \%$ of gynecological admissions. Cervical cancer constituted $58.9 \%$ (139/236) of all gynecological malignancies. One hundred and six cases of cervical cancer for which case records were available were analyzed. The mean age of women with cervical cancer was 57.5 \pm 12.6 (range 35-82) years, and approximately 69\% (73/106) of women with cervical cancer were over 50 years of age, 92\% (98/106) were multiparous, and 75\% (80/106) were postmenopausal. Table 2 shows the clinicopathological characteristics of cervical cancer during the study period. All but one cervical cancer patients had examination under anesthesia, clinical 
Table I Distribution of gynecological cancers based on characteristics of patients

\begin{tabular}{|c|c|c|}
\hline Characteristic & $\begin{array}{l}\text { Frequency of gynecological } \\
\text { cancer }(n=200)\end{array}$ & Percentage \\
\hline \multicolumn{3}{|l|}{ Age (years) } \\
\hline $10-20$ & 5 & 2.5 \\
\hline $21-30$ & 11 & 5.5 \\
\hline $31-40$ & 16 & 8.0 \\
\hline $4 I-50$ & 40 & 20.0 \\
\hline $51-60$ & 47 & 23.5 \\
\hline $6 I-70$ & 49 & 24.5 \\
\hline $7 I-80$ & 22 & II \\
\hline \multicolumn{3}{|l|}{ Parity } \\
\hline 0 & 27 & 13.5 \\
\hline $1-4$ & 96 & 48.0 \\
\hline$\geq 5$ & 67 & 33.5 \\
\hline \multicolumn{3}{|l|}{ Marital status } \\
\hline Single & 21 & 10.5 \\
\hline Married & 109 & 54.5 \\
\hline Widowed & 60 & 30.0 \\
\hline \multicolumn{3}{|l|}{ Religion } \\
\hline Christianity & 184 & 92.0 \\
\hline Islam & 5 & 2.5 \\
\hline Traditional religion & 1 & 0.5 \\
\hline \multicolumn{3}{|c|}{ Christian denomination } \\
\hline Roman Catholic & 78 & 39.0 \\
\hline Anglican & 47 & 23.5 \\
\hline Pentecostal & 53 & 26.5 \\
\hline Others & 8 & 4.0 \\
\hline \multicolumn{3}{|l|}{ Ethnic group } \\
\hline Igbo & 178 & 89.0 \\
\hline Non-lgbo & 12 & 6.0 \\
\hline \multicolumn{3}{|l|}{ Occupation } \\
\hline Unemployed & 71 & 35.5 \\
\hline Farmer & 40 & 20.0 \\
\hline Civil servant & 21 & 10.5 \\
\hline Trader & 35 & 17.5 \\
\hline Teacher & 5 & 2.5 \\
\hline Nurse & 2 & 1.0 \\
\hline Student & 16 & 8.0 \\
\hline \multicolumn{3}{|l|}{ Menstrual status } \\
\hline Premenopausal & 57 & 28.5 \\
\hline Post-menopausal & 133 & 66.5 \\
\hline
\end{tabular}

staging, and biopsy. Approximately $94.3 \%$ (100/106) of cases presented in stages 3 and 4; 4.7\% (5/106) presented in stage 2 , while $0.9 \%(1 / 106)$ was diagnosed after a cone biopsy for cervical intraepithelial neoplasia type III and was in stage $1 b$.

Treatment of patients with stages 3 and 4 cervical cancer involved mainly symptomatic care/palliative care with injectable and/or oral analgesics, antibiotics, blood transfusion, and intravenous infusions. There was no record of patientcontrolled analgesia for cervical cancer. Two cases with stage $3 \mathrm{~b}$ disease had urinary diversion (suprapubic cystostomy) by urologists to relieve bladder outlet obstruction.
Two of the five cases with stage 2 disease were in stage $2 \mathrm{a}$ and had radical hysterectomy/bilateral salpingooophorectomy: the other three were in stage $2 b$ and were referred directly for radical radiotherapy. Referrals for radiotherapy were to any of the four centers with functional radiotherapy facilities in the country during the study period. There was no radiotherapy facility in the study center during the study period. The only case with stage $1 \mathrm{~b}$ disease had simple hysterectomy and bilateral salpingo-oophorectomy.

Approximately $56.6 \%(60 / 106)$ of the patients were discharged home on request to continue palliative care with appointments to return for further review, and all were lost to follow-up. The only case with stage $1 \mathrm{~b}$ disease had a record of a hospital visit 2 years after hysterectomy and had remained symptom-free. She was subsequently lost to follow-up. Approximately 39.6\% (42/106) of cervical cancer patients had a record of death while still in the hospital, and these included the two who had radical hysterectomy and died within one week of surgery. Mortality and survival data for the remaining $60 \%$ were not available.

\section{Ovarian cancer}

There were 54 cases of ovarian cancer, constituting $0.8 \%$ of all gynecological admissions. The records of 50 cases of ovarian cancer were available for analysis. Ovarian cancer constituted $22.9 \%$ (54/236) of all gynecological malignancies. The mean patient age for all histological subtypes was $45.4 \pm 17.1$ years. The mean patient ages for the different subtypes were epithelial cancer $50.3 \pm 13.2$ years, sex cord $53.1 \pm 13.3$ years, and germ cell $18.5 \pm 8.3$ years. Table 2 shows the clinicopathological characteristics of ovarian cancer during the study period.

Women who had ovarian cancer were on average younger than those with other gynecological cancers (mean age 45.4 years versus 57.4 years; $P<0.001$ ). For epithelial ovarian cancer specifically, the average age at presentation was also significantly lower than the average age of presentation for the other primary gynecological cancers ( 50.3 years versus 57.4 years; $P=0.03$ ). Most cases of epithelial ovarian cancer occurred at 50 years or younger, and epithelial ovarian cancer was more prevalent under 50 years compared with other gynecological cancers ( $52 \%$ versus $30 \%, P=0.01$ ). Multiparous women constituted $72.40 \%$ (including 38\% in grand multiparous women) of epithelial ovarian cancer, and about $35 \%$ of epithelial ovarian cancers occurred in premenopausal women.

Management of ovarian cancer was through a multidisciplinary approach involving gynecologists, general 
Table 2 Clinicopathological features of cervical and ovarian cancers seen at the University of Nigeria Teaching Hospital, Enugu, from 2000 to 2010

\begin{tabular}{|c|c|c|c|c|}
\hline Type of cancer & Clinical/pathological features & & Cases (n) & Percentage \\
\hline \multirow[t]{9}{*}{ Cervical $(n=106)$} & Presenting symptoms & Post-menopausal bleeding & 105 & 99.1 \\
\hline & & Foul-smelling vaginal discharge & 70 & 66.0 \\
\hline & & Post-coital bleeding & 33 & 31.1 \\
\hline & Stage of disease & 1 & I & 0.9 \\
\hline & & 2 & 5 & 4.7 \\
\hline & & 3 & 61 & 57.5 \\
\hline & & 4 & 39 & 36.8 \\
\hline & Histological type & Squamous carcinoma & 101 & 95.3 \\
\hline & & Adenocarcinoma & 5 & 4.7 \\
\hline \multirow{17}{*}{ Ovarian $(n=50)$} & Presenting symptoms & Abdominal mass & 32 & 64 \\
\hline & & Abdominal pains and discomfort & 26 & 52 \\
\hline & & Weight loss & 6 & 12 \\
\hline & Stage of disease at & 1 & 0 & 0 \\
\hline & presentation & 2 & 4 & 8 \\
\hline & & 3 & 32 & 64 \\
\hline & & 4 & 14 & 28 \\
\hline & Histological type & Subtype & & \\
\hline & Epithelial tumors & Serous cystadenocarcinoma & 18 & 36 \\
\hline & & Mucinous cystadenocarcinoma & 12 & 24 \\
\hline & & Poorly differentiated adenocarcinoma & 4 & 8 \\
\hline & Sex cord stromal tumors & Granulosa cell tumor & 4 & 8 \\
\hline & & $\begin{array}{l}\text { Poorly differentiated Sertoli-Leydig } \\
\text { cell tumor }\end{array}$ & 2 & 4 \\
\hline & & Invasive alveolar rhabdomyosarcoma & 2 & 4 \\
\hline & Germ cell tumors & Malignant teratoma & 4 & 8 \\
\hline & & Dysgerminoma & 2 & 4 \\
\hline & & Endodermal sinus tumor & 2 & 4 \\
\hline
\end{tabular}

surgeons, and occasionally urologists; there was no trained gynecological oncologist in the center during the study period. General surgeons participated in most surgeries involving bowel resection. Most patients had optimal debulking surgery and chemotherapy. Approximately $76 \%$ (38/50) of the patients had surgical staging/biopsy and maximal debulking surgery involving total abdominal hysterectomy, bilateral salpingooophorectomy, and different combinations of omentectomy, appendectomy, bowel resection, and adhesiolysis.

The remaining 24\% (12/50) were treated as follows: one patient, a 9-year-old girl, had excision of a unilateral tuboovarian mass which was found to be a borderline tumor on histology. Four of the patients $(4 / 50,8 \%)$ were referred to the study center for histological diagnosis and chemotherapy after surgery had been done in private clinics. Seven of the 50 patients $(14 \%)$ had inoperable tumors at surgery; two of these had neoadjuvant chemotherapy with cisplatin and paclitaxel prior to surgery. All seven had staging/biopsy followed by palliative treatment.

Eighty-four percent (42/50) of the patients had chemotherapy involving cisplatin-based combination chemotherapy. The combinations comprised a platinum agent (cisplatin or carboplatin) and paclitaxel, cyclophosphamide, or doxorubicin. Approximately 74\% (37/50) of patients had cisplatin and cyclophosphamide or doxorubicin: only $10 \%(5 / 50)$ of patients were given combinations containing carboplatin or paclitaxel. However, approximately $81 \%$ (34/42) of patients who were given chemotherapy had one or two courses only and abandoned further chemotherapy. Approximately 28.6\% (6/42) had six courses, while $4.8 \%$ (2/42) had four courses. All patients had injectable and/ or oral analgesics for pain relief and antibiotics for sepsis as part of symptomatic/palliative therapy. The use of patient-controlled analgesia was not found in the records. Follow-up of patients was by clinical examination and transabdominal/transvaginal ultrasonography. CA125 assay was not routinely done because of lack of facilities within the hospital. Only two patients who had stage 2 disease had records of CA125 assays done outside the study center. Both were serous cystadenocarcinoma. Approximately $70 \%$ $(38 / 54)$ of cases had a record of death from the disease in the hospital; $63 \%$ of these (24/38) died within one week of 
surgery. Mortality or survival data for the remaining 29.6\% $(16 / 54)$ were not available.

\section{Corpus uteri cancer}

There were 33 cases of corpus uteri cancer, constituting $0.5 \%$ of all gynecological admissions. Corpus uteri cancers comprised approximately 14\% (33/236) of all gynecological malignancies. These cancers were made up of $19(57.6 \%)$ cases of endometrial carcinoma, ten $(30.3 \%)$ of choriocarcinoma, and four (12.1\%) of leiomyosarcoma. Table 3 shows the clinicopathological features of corpus uteri cancer seen during the period.

Endometrial cancers constituted 8.1\% (19/236) of cases of all gynecological malignancies. The mean age of patients with endometrial carcinoma was 56.1 \pm 12.7 years. Approximately 90\% (17/19) of cases were multiparous. Approximately $90 \%$ of cases of endometrial carcinoma presented in stages 1 and 2 . The commonest presenting symptoms for endometrial cancer were postmenopausal bleeding (78.3\%) and lower abdominal pain (26\%). Endometrial cancer was not recorded in patients under the age of 50 years. All cases of endometrial carcinoma had an initial endometrial biopsy and definitive interval surgery. Facilities for histological diagnosis by frozen section were not available. Surgery involved total abdominal hysterectomy and bilateral salpingo-oophorectomy with or without pelvic lymphadenectomy. Adjunctive treatment with progestogens was given to two patients $(2 / 10 ; 10 \%)$ with stage 3 and 4 disease. No follow-up data beyond 3 months were available for the patients who had surgery. No mortality data were available for endometrial cancer.

Choriocarcinoma constituted 4.2\% (10/236) of all gynecological malignancies. The mean age of patients with

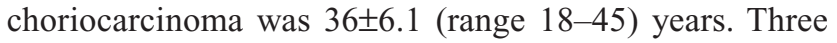
cases $(30 \%)$ followed term pregnancies, two $(20 \%)$ followed abortions, while five (50\%) followed molar pregnancies. The commonest presenting complaints were amenorrhea $(100 \%$, $10 / 10)$, followed by vaginal bleeding $(90 \%, 9 / 10)$, and lower abdominal pain $(60 \%, 6 / 10)$. Approximately $10 \%$ were in stage $1,70 \%$ in stage 2 , and $10 \%$ each in stages 3 and 4 : the only stage 4 case had pulmonary metastasis. In all cases, diagnosis involved assays of serum beta-human chorionic gonadotropin. Chemotherapy was the mainstay of treatment for all patients. Twenty percent $(2 / 10)$ of cases had total abdominal hysterectomy for heavy uncontrollable vaginal bleeding. Chemotherapy regimens used included methotrexate, cyclophosphamide, cisplatin, and doxorubicin in combination. There was a record of death for two patients while in hospital, giving a case fatality rate of $20 \%$.

Leiomyosarcoma constituted 1.7\% (4/236) of all gynecological malignancies. The mean age of cases of leiomyosarcoma was $58.3 \pm 11.5$ years. Three cases presented with lower abdominal masses, excessive menstrual flow, and lower abdominal pain, and were diagnosed after histology for uterine fibroids removed during myomectomy and were classified as stage 1 tumors. These patients subsequently underwent total

Table 3 Clinicopathological features of corpus uteri, vulvar, and vaginal cancers seen at the University of Nigeria Teaching Hospital, Enugu, from 2000 to 2010

\begin{tabular}{|c|c|c|c|c|}
\hline \multicolumn{3}{|l|}{ Cancer type } & \multirow{2}{*}{$\begin{array}{l}\text { Number } \\
29\end{array}$} & \multirow{2}{*}{$\begin{array}{l}\text { Percentage } \\
87.9\end{array}$} \\
\hline Corpus uteri cancer & Presenting symptoms & Vaginal bleeding & & \\
\hline \multirow[t]{9}{*}{$(n=33)$} & & Lower abdominal mass & 6 & 18.1 \\
\hline & & Lower abdominal pains & 14 & 42.4 \\
\hline & Stage of disease & 1 & 3 & 8.7 \\
\hline & & 2 & 23 & 69.6 \\
\hline & & 3 & 6 & 21.7 \\
\hline & & 4 & 1 & 3 \\
\hline & Histological type & Endometrial carcinoma & 19 & 57.6 \\
\hline & & Choriocarcinoma & 10 & 30.3 \\
\hline & & Leiomyosarcoma & 4 & 12.1 \\
\hline \multirow[t]{8}{*}{ Vulvar $(n=10)$} & Presenting symptoms & Vulvar ulcer & 8 & 80 \\
\hline & & Vulvar itching & 2 & 20 \\
\hline & & Vulvar bleeding & 6 & 60 \\
\hline & Stage of disease & 1 & 2 & 20 \\
\hline & & 2 & 3 & 30 \\
\hline & & 3 & 4 & 40 \\
\hline & & 4 & 1 & 10 \\
\hline & Histological type & Squamous carcinoma & 10 & 100 \\
\hline
\end{tabular}


abdominal hysterectomy and bilateral salpingo-oophorectomy. The remaining patient had a large lower abdominal mass and severe pain. An inoperable stage 4 tumor was found at laparotomy. The patient had palliative treatment with injectable and oral analgesics, hematinics (excluding folic acid) and antibiotics. Records showed no evidence that any of the cases ever returned after surgery. No mortality or survival data were available for any of the four cases.

\section{Vulvar cancer}

There were ten cases of vulvar cancer, constituting $0.15 \%$ of all gynecological admissions and $4.2 \%$ of all gynecological malignancies. The mean age of cases was $58.7 \pm 4.9$ (range 52-85) years. The clinicopathological characteristics of the vulvar cancer seen during the study period are summarized in Table 3. In addition to analgesia, antibiotics, and hematinics, patients also had simple or radical vulvectomy and inguinal lymphadenectomy with or without referral for radiotherapy. There was no record of death in the hospital from vulvar cancer. All patients were available for follow-up for not more than 6 months, with two referred for radiotherapy. All patients were apparently lost to follow-up.

\section{Discussion}

This study describes the management of gynecological cancers in a major referral hospital for cancer patients in South East Nigeria. In particular, the study highlights the major challenges to gynecological cancer management at the center during the study period.

The study found that the most common gynecological cancers (cervical and ovarian) presented in advanced stages of the diseases, and too late for curative interventions to be undertaken. For instance, most patients who had cervical cancer were offered symptomatic treatment because they presented in advanced stages of the disease. Late presentation is a major challenge to successful treatment of cervical and ovarian cancers in this environment. The late presentation of cervical cancer patients found in this study agrees with previous studies in Nigeria. ${ }^{10-12}$ Based on earlier reports and anecdotal evidence, late presentation of cervical cancer in developing countries appears to be a multifactorial phenomenon. One factor responsible for this trend is the inability of patients to detect early stages of the disease. This has been ascribed to the absence or poor uptake of the few screening services available. ${ }^{13}$ Nationally organized screening programs such as those implemented in most Western countries offer the best approach to wider coverage of the population. ${ }^{13}$ Such national screening programs integrate screening into the health care system and tend to make the population more conscious of the need for screening. ${ }^{13}$ It is well known that cervical cancer screening not only detects precancerous stages of the disease, but can also detect early stage cancers when cure is theoretically possible. ${ }^{14}$

Other possible causes of late presentation of gynecological cancers are related to the societal illness behavior in developing countries and the socioeconomic status of women. The illness behavior of many African societies, for instance, is still pervaded by ignorance and rooted in traditional belief systems despite apparent exposure to Western civilization. ${ }^{5}$ One such belief is that diseases such as cancer are caused by witchcraft or spiritual poisoning by perceived enemies of the patient and that certain diseases are not amenable to orthodox medical care. ${ }^{5}$ Even when symptoms of early disease are present, many patients would tend to go to spiritualists and herbalists first before seeking orthodox care. Added to these erroneous beliefs is the fact that herbalists offer cheaper care than hospitals and many poor patients would seek cheaper care from herbalists first and resort to orthodox treatment only when this cheaper care fails to give the desired relief. ${ }^{6}$ Anecdotal evidence suggests that the subordinate roles of women in traditional African societies limit the capacity of women to express themselves, including reporting physical symptoms related to the genital tract or seeking medical attention without the approval of the husband or the husband's family. Related to the subordinate role of women is the economic disempowerment of women, which results in a lack of financial ability to fund treatment. All these factors contribute to delays in presentation for care in hospital.

With respect to ovarian cancer, late presentation is partly due to the anatomical location of the organ, which is responsible for the nonspecific symptoms of early ovarian cancer. ${ }^{15}$ Further, early ovarian cancer can be asymptomatic. In the absence of any known premalignant phase of the disease and lack of any effective screening test, the best hope for early detection of ovarian cancer lies with a high index of suspicion facilitated by a high degree of health awareness. ${ }^{16}$ Such a level of health awareness may not be attainable in many developing countries.

Another finding in this study that represents a formidable hindrance to successful outcomes of treatment for gynecological cancer is the inadequacy, and in some cases nonavailability, of facilities and expertise for proper diagnosis and treatment. This agrees with previous reports from developing countries. ${ }^{5,17}$ Cancer care is hardly a priority for scarce health care resources in most developing countries as they battle formidable challenges from infectious diseases such as 
malaria, human immunodeficiency virus, and tuberculosis. In this study, for instance, functioning radiotherapy facilities were not available in the center during the study period. There was no evidence of a trained gynecological oncologist, medical oncologist, or oncology nurses throughout the study period. All those referred to other centers for radiotherapy needed to travel hundreds of kilometers to any of the three cities where radiotherapy facilities were available. Also, CA125 assays could not be done in the center. Cutting edge cytotoxic drugs were not used for chemotherapy, probably because the latest cytotoxic drugs used in Western countries are generally not available or affordable in developing countries. Lack of facilities meant that even when patients with the disease presented early, the full complement of care possible could be unavailable. The high postoperative mortality for ovarian and cervical cancers could be attributable, at least in part, to a lack of appropriate surgical skills and limited intensive care facilities in the center. All these factors clearly militate against successful treatment of gynecological cancer.

Although developments in the last 2 years in the center, such as creation of a gynecological oncology unit and the commencement of some radiotherapy services are positive developments, empirical observation suggests that trained personnel are still very few. There is only one trained gynecological oncologist and one medical oncologist to serve the entire hospital. There are no trained oncology nurses, and intensive care facilities are limited. Although the cancer workload appears small, it is expected that the gynecological oncologist will be able to attract more gynecological cancer patients. This expected increase will help to maintain the skill set of the gynecological oncologist. There still exists a huge unmet need for trained medical oncologists, oncology nurses, radiotherapists, and interventional radiologists. Further, the full complement of radiotherapy for cervical cancer is still unavailable, given that the components required for brachytherapy are not yet installed.

A rather peculiar barrier to successful outcome of cancer care was the finding that many patients abandoned their treatment half-way, in particular chemotherapy. From previous reports, abandonment of treatment could be ascribed to several possible factors, including inability to sustain funding for treatment and inability to source prescribed drugs. ${ }^{5}$ Also, from anecdotal evidence, it could also be due to disenchantment with therapy due to the lack of clinical improvement following initial treatment or due to inability of patients to tolerate the side effects of cytotoxic drugs. Supportive care for the side effects of chemotherapy could have been hampered by the high cost or lack of cutting edge antiemetics, erythropoietin, and blood transfusion facilities. Abandonment of treatment can be challenging for the care team because it denies them the opportunity to evaluate prescribed treatments and could lead to a sense of frustration and loss of enthusiasm on the part of the care team. Fortunately, most of the postulated factors that lead to abandonment of treatment are modifiable. Whereas the side effects of cytotoxic drugs or perception of clinical progress on the part of patients can be addressed by the managing clinicians through appropriate counseling and use of supportive treatment measures, the high cost and relative unavailability of many cutting edge cytotoxic drugs requires intervention by the government. There is a need to subsidize the cost of these drugs to make them affordable for most of those who need them. Unfortunately, the medical insurance scheme evolving in Nigeria does not include cancer care at present, so patients have to pay out of pocket.

The major strength of this study was the use of a retrospective longitudinal analytical technique that is suitable for evaluation of the presentation and follow-up of management and outcomes of rare diseases like cancer. The major limitations relate to the small number of gynecological cancers treated in the center, suggesting that many gynecological cancer patients probably did not seek treatment at the center. There is a possibility of a referral bias arising from the societal perception that cancer is not curable by orthodox medicine. The small sample size limits the external validity of the findings of this study. Another limitation was the inability to obtain records for all gynecological cancer patients seen in the center, as well as the absence of comprehensive mortality and survival data for all patients. These limit the interpretation of the presented data and also the internal validity of the study findings.

In conclusion, the major challenges to a successful outcome of treatment for gynecological cancer at this center include the late presentation of most cases, poor outcomes of complex gynecological cancer surgery, noncompliance with prescribed treatment regimens, and inadequate facilities for radiotherapy. It is recommended that government should subsidize gynecological cancer care to reduce the cost of investigation and treatment, and perhaps improve compliance with treatment regimens. More training is needed in gynecological cancer surgery for gynecologists in this center and specifically more trained gynecological oncologists are needed. Behavioral change in communication techniques should be deployed through public health campaigns to educate the public on the need to use the 
few available cancer screening services and to present early for treatment, so as to improve the prognosis of these diseases.

\section{Acknowledgments}

We acknowledge the contribution of Dr OC Okafor at the University of Nigeria Teaching Hospital for providing the anonymized histopathological diagnoses of gynecological cancer patients and the staff of the records department and cancer registry for their assistance with patient records.

\section{Disclosure}

The authors report no conflicts of interest in this work.

\section{References}

1. The Economist Intelligence Unit. Breakaway: the global burden of cancer - challenges and opportunities. The Economist. 2009. Available from: https://assets-livestrong-org.s3.amazonaws.com/media/site_proxy/data/ c49ced3068f7205319cb1edf653dd91e0baee3ba.pdf. Accessed December 12, 2013.

2. Ferlay J, Bray F, Norman D, Mathers C, Parkin DM. GLOBOCAN 2008, Cancer Incidence and Mortality Worldwide. Lyon, France: International Agency for Research on Cancer; 2008.

3. Wilson CM, Tobin S, Young RC. The exploding worldwide cancer burden: the impact of cancer on women. Int J Gynecol Cancer. 2004;14:1-11.

4. Iyoke CA, Ugwu GO. Burden of gynaecological cancer in developing countries. World J Obstet Gynaecol. 2013;2:1-7.
5. Price AJ, Ndom P, Atenguena E, Mambou Nouemssi JP, Ryder RW. Cancer care challenges in developing countries. Cancer. 2012;118: 3627-3635.

6. International Federation of Gynaecology and Obstetrics. 26th annual report on the results of treatment of gynaecological cancer. Int $J$ Gynaecol Obstet. 2006;95 Suppl 1:S161-S191.

7. Ugwu VEO, Iferikigwe ES, Okeke TC, Ugwu AO, Okezie OA, Agu PU. Pattern of gynaecological cancers in the University of Nigeria Teaching Hospital, Enugu, South East Nigeria. Niger J Med. 2011;20:266-269.

8. Okeke T, Onah N, Ikeakor L, Ezenyeaku C. The frequency and pattern of female genital tract malignancies at the University of Nigeria Teaching Hospital, Enugu, Nigeria. Ann Med Health Sci Res. 2013;3:345-348.

9. Anya SE, Ezugwu FO, Okaro JM. Gynaecologic mortality in Enugu, Nigeria. Trop Doct. 2006;36:235-236.

10. Mohammed A, Ahmed SA, Oluwole OP, Avidime S. Malignant tumours of the female genital tract in Zaria, Nigeria: analysis of 513 cases. Ann Afr Med. 2006;5:93-96.

11. Bassey EA, Ekpo MD, Abasiatai A. Female genital malignancies in Uyo, South-South Nigeria. Niger Postgrad Med J. 2007;14:134-136.

12. Anorlu RI, Obodo K, Makwe CC. Cancer mortality among patients admitted to gynecological wards at Lagos University Teaching Hospital, Nigeria. Int J Gynaecol Obstet. 2010;110:268-269.

13. Denny L. Cytological screening for cervical cancer prevention. Best Pract Res Clin Obstet Gynaecol. 2012;26:189-196.

14. Shepherd JH. Cervical cancer. Best Pract Res Clin Obstet Gynaecol. 2012;26:293-309.

15. Goff B. Symptoms associated with ovarian cancer. Clin Obstet Gynecol. 2012;55:36-42.

16. Hennessy BT, Coleman RL, Markman M. Ovarian cancer. Lancet. 2009;374:1371-1382.

17. Basile S, Angioli R, Mana N, Palaia I, Plotti F, Panici PB. Gynaecological cancers in developing countries: the challenge of chemotherapy in low resource setting. Int J Gynaecol Cancer. 2006;16:1491-1497.
International Journal of Women's Health

\section{Publish your work in this journal}

The International Journal of Women's Health is an international, peerreviewed open-access journal publishing original research, reports, editorials, reviews and commentaries on all aspects of women's healthcare including gynecology, obstetrics, and breast cancer. The manuscript management system is completely online and includes

\section{Dovepress}

a very quick and fair peer-review system, which is all easy to use. Visit http://www.dovepress.com/testimonials.php to read real quotes from published authors. 